\title{
Review of Different Local and Global Contrast Enhancement Techniques for a Digital Image
}

\author{
Shefali Gupta \\ Research Scholar \\ Chandigarh Group of Colleges \\ Gharuan, Mohali
}

\author{
Yadwinder Kaur \\ Associate Professor \\ Chandigarh University \\ Gharuan, Mohali
}

\begin{abstract}
Image enhancement is one of the challenging issues in low level image processing. The main aim of image enhancement is to enhance quality of the image so that visual appearance can be improved. Contrast enhancement is an important factor for image enhancement. Histogram based techniques are one of the most important image processing techniques that are used for enhancement tasks. Histogram equalization is a very effective approach to contrast enhancement. However, histogram equalization tends to change the brightness of the image. The present paper describes a review of different local and global contrast enhancement techniques for a digital image.
\end{abstract}

\section{Keywords}

Image, Contrast Enhancement, Histogram Equalization, BBHE, Adaptive Histogram Equalization

\section{INTRODUCTION}

A digital image $f(x, y)$ is a two-dimensional function where $x \& y$ are spatial coordinates and amplitude of $f$ at any pair of coordinates $(x, y)$ is called intensity or gray level of image of that point. Digital image processing is a composition of a finite number of elements called picture elements, image elements, pels or pixels [2]. Image enhancement is one of the challenging issues in low level image processing. It is a process of manipulating the image such that resulting image is more suitable than the original one. The principle aim of image enhancement lies in improving the quality of image to make the image better in visual appearance. The interpretability of information in images is also improved for human viewers and a better input can be provided for other automated image processing techniques. Generally image enhancement techniques are used to highlight certain features of interest in the image. To improve the visual quality of images, image enhancement techniques provide a variety of ways like removing unwanted noise, improving contrast, deblurring, smoothing, sharpening, and edge enhancement etc [23]. Image enhancement has contributed to research advancement in a variety of fields like medical image analysis, high definition television (HDTV), industrial X-ray image processing, microscopic imaging, remote sensing etc.

Digital image processing is a broad subject that involves mathematically complex procedures, but central idea behind image processing is very simple [22]. In the image enhancement process, an image is taken as input and enhancement algorithm is applied on it. After that enhanced image is taken as output as shown in figure1.

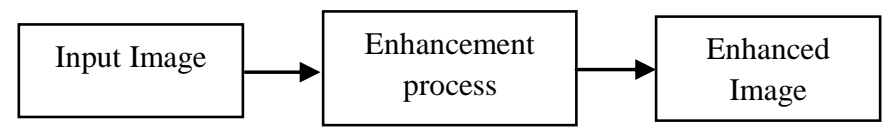

Fig. 1 Basic enhancement process

Image enhancement can be subjective or objective. Subjective image enhancement can be repeatedly applied on an image in many forms until the observer feels that output image yields the required necessary details. Objective enhancement process is not repeatedly applied but corrects an image for some known degradations and hence this enhancement is not applied arbitrarily [24].

\section{CONTRAST ENHANCEMENT TECHNIQUES}

The commonly used techniques for image enhancement are removal of noise, edge enhancement and contrast enhancement. Out of these contrast enhancement is a popular one. Contrast enhancement is one of the most important techniques for image enhancement [1]. In this technique, contrast of an image is improved to make the image better for human vision. The term contrast, as observed in digital images, is the separation of dark and bright areas present in the image. One of the most common contrast enhancement methods is histogram equalization (HE). This paper presents a review of various techniques that can be used for contrast enhancement. These techniques are categorized as global enhancement techniques and local enhancement techniques. Global techniques are fast and simple, and are suitable for overall enhancement of the image. These techniques cannot adapt to local brightness features of the input image because only global histogram information over the whole image is used [25]. This fact limits the contrast ratio in some parts of the image and hence causes significant contrast losses in the background and other small regions. One such example of global enhancement technique is global histogram equalization.

Several other local enhancement techniques are also used. Local enhancement techniques can enhance overall contrast more effectively. In local enhancement, a small window slides through every pixel of the input image sequentially and only those block of pixels are enhanced that fall in this window. And then gray level mapping is done only for the center pixel of that window [21]. Thus, it makes good use of local information. However, in local enhancement techniques, computational cost goes very high due to its fully overlapped sub-blocks and causes over-enhancement in some portions of the image. Another problem is that it enhances the noise effect in the image as well. 
Various global techniques for contrast enhancement are Brightness Bi-Histogram Equalization (BBHE), Dualistic Subimage Histogram Equalization (DSIHE), Recursive Mean Separate Histogram Equalization (RMSHE), Minimum Mean Brightness Error Bi-HE (MMBEBHE), Recursive Separated and Weighted Histogram Equalization (RSWHE) etc [10]. Local techniques include Adaptive Histogram Equalization (AHE), Contrast Limited Adaptive Histogram Equalization (CLAHE) etc.

The rest of the paper is organized as follows: Histogram equalization (HE) for a digital input image is reviewed with its mathematical formulation in section III; other global enhancement techniques like BBHE, DSIHE, RMSHE (generalization of BBHE), MMBEBHE, and RSWHE are discussed in section IV. In section V, some local enhancement techniques like AHE and CLAHE etc. are discussed. Paper concludes with Section VI containing discussion of various histogram equalization techniques.

\section{HISTOGRAM EQUALIZATION}

Histogram equalization (HE) is a widely used technique for contrast enhancement because it is simple to use and better in performance on all types of images. It is most commonly used in the areas like medical image processing, radar signal processing etc. HE works by flattening the histogram of input image and stretches dynamic range of gray levels by using cumulative density function (CDF) of the image. Histogram of an image represents the relative frequency of occurrence of gray levels to preserve mean brightness of the input image [3]. The HE method re-maps the gray levels of input image by reassigning intensity values of pixels to make a uniform intensity distribution. However, histogram equalization possesses some drawbacks. First, histogram equalization transforms histogram of original input image into a flat histogram where mean value lies somewhere in middle of gray level range, i.e. mean brightness of output image almost lies at the middle. Hence it does not take into account mean brightness of input image. Second, the HE method performs enhancement based on global content, i.e. it enhances borders and edges among objects in the image but local enhancement is negligible. Third, HE may result in over enhancement due to stretching of the gray levels of input image over the full gray level range [10].

For a given image $X=\{X(i, j)\}$, composed of $L$ discrete gray levels denoted as $\left\{X_{0}, X_{1}, \ldots X_{L-1}\right\}$, where $X(i, j)$ represents an intensity of image at the spatial location $(i, j)$ and $X(i, j) \in\left\{X_{0}, X_{1}, \ldots X_{L-1}\right\}$. For image $X$, probability density function $p\left(X_{K}\right)$ is defined as

$$
p\left(X_{K}\right)=\frac{n^{k}}{n}
$$

for $k=0,1, \ldots, L-1$, where $n^{k}$ represents number of times $X_{k}$ appears in input image $X$ and $n$ is total number times of samples in input image.

Here $p\left(X_{K}\right)$ is associated with histogram of input image which represents number of pixels having specific intensity $X_{k}$. A plot of $n^{k}$ vs. $X_{k}$ is known as histogram of $X$.
The cumulative density function (CDF) $c(x)$ is defined on the bases of PDF,

$$
c(x)=\sum_{j=0}^{k} p\left(X_{j}\right)
$$

where $X_{k}=x, \quad$ for $\quad k=0,1, \ldots, L-1 . \quad$ Here $c\left(X_{L-1}\right)=1$ by definition. HE is a scheme which maps input image into the entire dynamic range, $\left(X_{0}, X_{L-1}\right)$ by using CDF as a transform function [3]. Fig2 (a) and (b) illustrates histogram equalization technique.
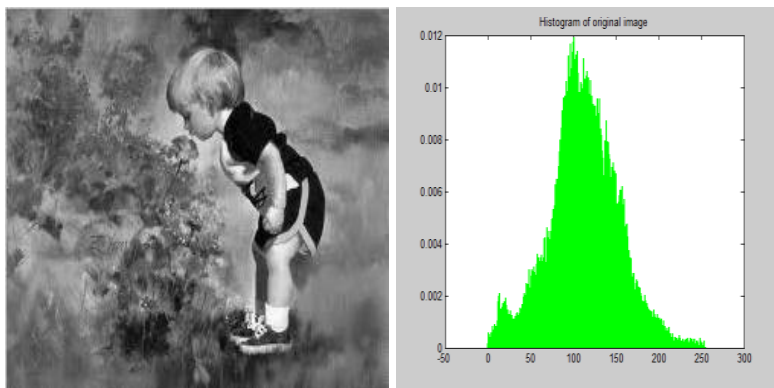

Fig. 2(a). Original gray scale image and its histogram

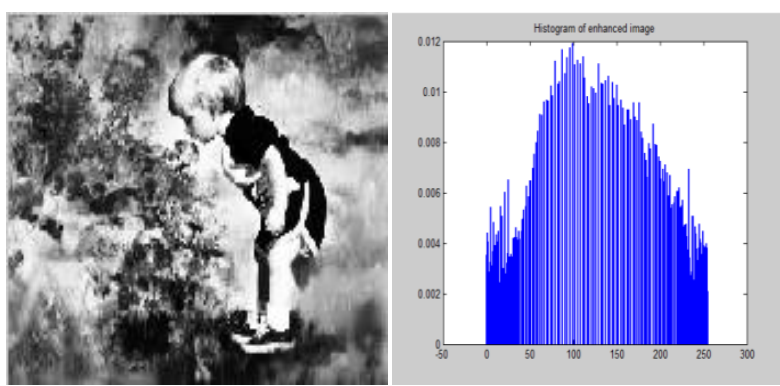

Fig. 2(b). Result of HE and its histogram

$\mathrm{HE}$ is the most common method used for contrast enhancement due to its simplicity. Some other disadvantage includes change in the brightness of image after $\mathrm{HE}$ is applied. Moreover, this technique is not commonly used in consumer electronics as it significantly changes brightness of input image and unnecessary visual deterioration is introduced [9] [13].

\section{GLOBAL ENHANCEMENT TECHNIQUES}

Global techniques are fast and simple, and are suitable for overall enhancement of the image. These techniques cannot adapt to local brightness features of the input image because only global histogram information over the whole image is used [25]. Some global techniques for contrast enhancement like BBHE, DSIHE, RMSHE, MMBEBHE, RWSHE etc. are explained in the following section.

\subsection{Brightness Preserving Bi-Histogram Equalization (BBHE):}

In this technique, the input image is decomposed and two sub images are formed on the bases of mean value. One subimage contains the set of samples that are less than or equal to mean whereas the other subimage is the set of samples greater than mean. Then the method equalizes both sub images independently according to their respective histograms with a 
constraint that samples in the first subimage are mapped in the range from minimum gray level to input mean and samples in second subimage are mapped in the range from mean to maximum gray level [10]. That means one subimage is equalized over the range up to mean and other subimage is equalized over the range from mean based on the respective histograms. The resultant equalized sub images are bounded by each other around input mean, which has an effect of preserving the mean brightness [1][3].

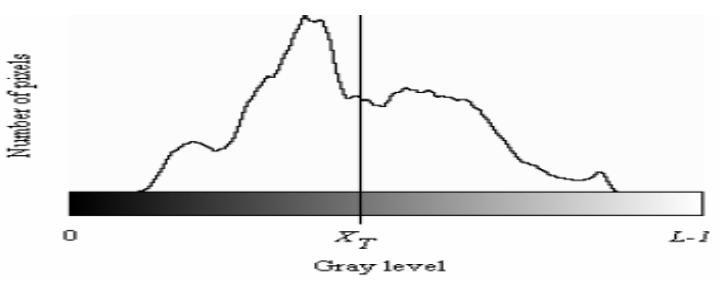

Fig. 3. Bi- Histogram Equalization

BBHE has an advantage that it preserves mean brightness of the image while enhancing the contrast and, thus, provides much natural enhancement that can be utilized in consumer electronic products [3].

\subsection{Dualistic Subimage Histogram Equalization (DSIHE):}

Some enhancement techniques change the luminance of image significantly with equalization. Such techniques can never be utilized in video systems directly. The DSIHE technique for contrast enhancement decomposes an image into two equal area sub-images, one dark and one bright, following the equal area property (i.e., both sub-images have same amount of pixels) $[10,14]$. This decomposition is done on the bases of its gray level cumulative probability density which is equal to 0.5. Then the two sub images are taken in equalization process respectively. After enhancement, these two sub images are composed into one image. Finally, result of enhancement provides an enhanced image with its original luminance that makes it possible to be used in the video system directly [11]. There is no doubt that these two sub images represent the dark and bright area of original image respectively. So, the gray level can be remained in its original scale respectively after subimage histogram equalization. Furthermore, contrast of the original image is also enhanced effectively post processing. The DSIHE method decomposes the images aiming at the maximization of the Shannon's entropy of the output image [1] [6].

\subsection{Recursive Mean-Separate Histogram Equalization (RMSHE):}

Mean-separation means to separate an image based on the mean of input image [7]. However, RMSHE technique is an extension of BBHE (where mean-separation was done only once). In RMSHE, instead of decomposing the input image only once, it is decomposed recursively up to a recursion level $r$, hence $2 r$ sub images are generated. Each subimage is then equalized independently with histogram equalization method. If $r=0$, that means no subimage decomposition is done, i.e. it is equivalent to HE method only [1][10]. When one mean separation is done before equalization, i.e. $r=1$, this is equivalent to BBHE [14]. This increases a level of brightness preservation. Similarly, two mean-separations before equalization will result in much higher level of brightness preservation as compared to $r=0$ and $r=1$ levels [7]. The above discussion concludes that the level of brightness preservation will increase with the increase of number of recursive meanseparations. This technique aims to bring more extends of brightness preservation than $\mathrm{HE}$ and BBHE techniques.

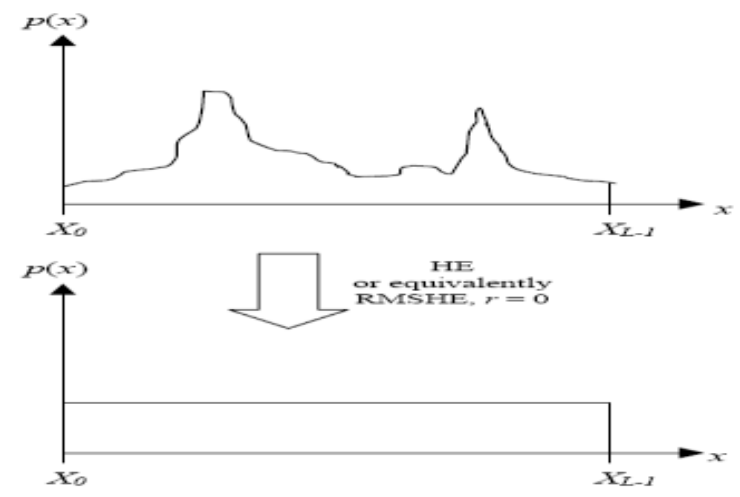

Fig. 4(a). Histogram before and after HE or equivalently RMSHE, $\mathbf{r}=\mathbf{0}$

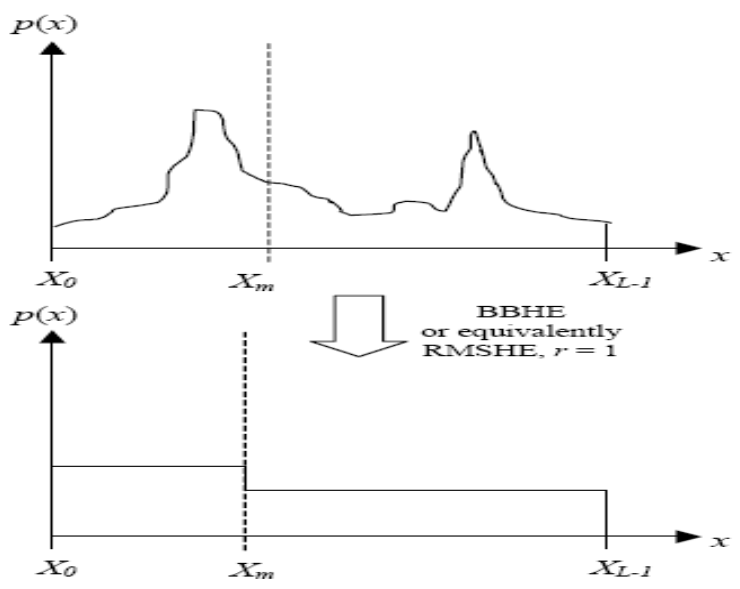

Fig. 4(b). Histogram before and after HE or equivalently RMSHE, $r=1$

\subsection{Minimum Mean Brightness Error $\mathrm{Bi}$ - Histogram Equalization (MMBEBHE):}

This technique, Minimum Mean Brightness Error BiHistogram Equalization, is based on the principle of Brightness Preserving Bi-Histogram Equalization (BBHE) and Dualistic Subimage Histogram Equalization (DSIHE), i.e. decomposition of image into two sub images and applying equalization process independently to the resulting sub images [1] [10]. But MMBEBHE is slightly different. This technique searches for a threshold level $l_{t}$, which decomposes input image into two sub-images in such a way that the minimum brightness difference between input image and output image is achieved. This is called absolute mean brightness error (AMBE) [15]. After decomposing input image by the threshold level, each of the two sub-images undergo histogram equalization process to generate the output image. The technique is summarized as follows:

a. Calculate the absolute mean brightness error (AMBE) for each possible threshold level.

b. Find a threshold level XT that yield minimum absolute mean brightness error (AMBE).

c. Separate the input histogram into two histograms based on $X_{T}$ found in Step 2 and equalize both the histograms independently [14]. 
This technique aims to produce a method that is suitable for real-time applications.

\subsection{Recursive Separated and Weighted Histogram Equalization (RSWHE):}

The RSWHE technique is slightly different from the techniques discussed so far in this section. The main difference between RSWHE and other histogram equalization techniques is that RSWHE first modifies the input histogram and then runs the equalization procedure. This technique works in three modules. These are: histogram segmentation, histogram weighting and histogram equalization [1][17].

The idea behind each module in RSWHE technique is explained as follows:

i) Histogram segmentation module It takes the input image, computes the input histogram. The input histogram is decomposed recursively into two or more sub-histograms based on the mean and median value [16].

ii) Histogram weighting module In this module, subhistograms computed in step 1 are modified through histogram weighting process using a normalized power law function.

iii) Histogram equalization module In this, histogram equalization process is individually applied over each of the weighted sub-histograms that were modified in step 2. A better contrast enhancement is achieved by equalizing each sub-histogram independently and annoying side effects are also reduced through RSWHE [1].

Recursive sub-image histogram equalization (RSIHE) and recursive mean separate histogram equalization (RMSHE) are some methods that are similar to RSWHE, but weighting process is not carried out in RSIHE and RMSHE.

\section{LOCAL ENHANCEMENT TECHNIQUES}

Local enhancement techniques enhance overall contrast more effectively than global techniques. Some local techniques for contrast enhancement like AHE, CLAHE etc. are explained in the following section.

\subsection{Adaptive Histogram Equalization (AHE):}

Adaptive histogram equalization is a method of contrast enhancement. It is different from ordinary histogram equalization. In adaptive method, many histograms are computed where each histogram corresponds to a different section of image. Hence, AHE improves the local contrast of an image and more details can be observed. With this method, information of all intensity ranges of the image can be viewed simultaneously. There are many ordinary display devices that are not able to depict the full dynamic intensity range. This method brings a solution to this problem. Other advantages include that it is automatic (i.e., no manual intervention is required) and reproducible from study to study.

In AHE method, for each pixel in the input image, a region centered about a pixel is assigned. This region is called contextual region (calculate size of contextual region before assigning) [26]. The intensities values for this region are used to find the histogram equalization mapping function and this mapping function is applied to the pixel being processed in the region. The resultant image is produced where each pixel is mapped differently. Hence the intensities are distributed locally and contrast is enhanced based on the local area rather than the entire image. Apart from the advantage of local enhancement, AHE method has some limitations also. This method works too slowly on a general purpose computer although it works correctly. As enhancement is carried out in a local area, AHE tends to over enhance the noise content.

\subsection{Contrast Limited Adaptive Histogram Equalization (CLAHE):}

CLAHE is locally adaptive contrast enhancement method. It is an improved version of adaptive histogram equalization method. The AHE technique has a practical limitation that homogenous regions of image can cause over-amplification of noise because a narrow range of pixels are mapped to an entire visualization range. Contrast limited AHE (CLAHE) was developed to prevent this over-amplification of noise in homogenous regions. CLAHE limits the amplification of noise in the image so that image appears more natural. This technique is also very useful for video broadcasting where brightness requirement is very high. CLAHE enhances brightness level to a specific range and hence facilitates the comparison of different areas of an image [21].

In CLAHE, the input image is partitioned into many non-overlapping regions that have almost equal sizes and $\mathrm{HE}$ is applied to each of this region. The obtained histograms for each region are clipped by a clip limit that is based on the desired contrast expansion and the size of the neighbourhood region. For each clipped histogram, a transformation function is obtained that performs gray scale mapping. Finally, CLAHE applies bilinear interpolation to eliminate the region boundaries [18]. Therefore small neighboring areas look smoother (as if no boundaries). The distribution of used gray levels becomes even by applying this technique and hence hidden features in the image become more visible

The advantages of using CLAHE are that it is easy to use, uses simple calculation, and gives good output in local areas of the image. CLAHE has less noise and it can prevent brightness saturation that commonly happens in histogram equalization [8].

\section{SIMULATION RESULTS}

Contrast enhancement of digital images was performed using local and global enhancement techniques. Histogram equalization (HE), Adaptive histogram equalization (AHE), Contrast limited adaptive histogram equalization (CLAHE) etc. were applied on input images. Analysis was made on the basis of some parameters like PSNR, MSE, MEAN etc.

\subsection{Enhancement using global techniques}

In this case, images can be enhanced globally, i.e. a better overall contrast enhancement is gained. Histogram equalization was applied on a digital image and output was observed as shown in figure 5 (a) and (b).

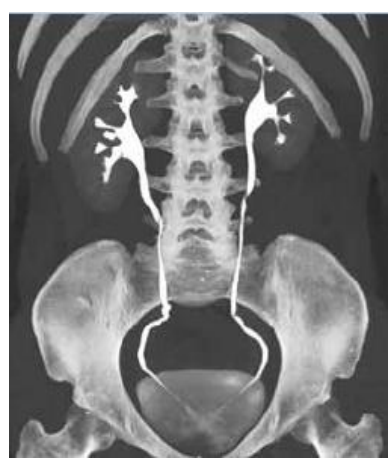

Fig. 5(a). Original image

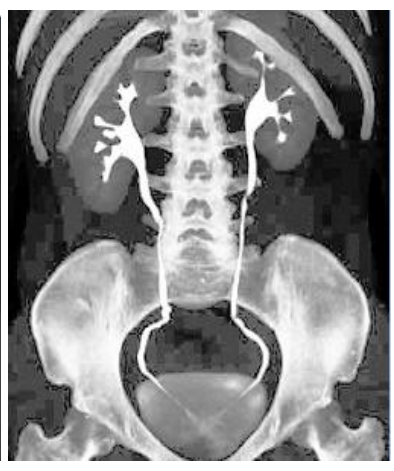

Fig. 5(b). HE image 


\subsection{Enhancement using local techniques}

In this case, images can be enhanced locally, i.e. much better contrast enhancement is achieved in local areas also. Adaptive histogram equalization (AHE), Contrast limited adaptive histogram equalization (CLAHE) etc. were applied on a digital image to observe the results in case of local techniques. The following fig 6(a) and 6(b) and fig 7(a) and 7(b) were observed when local techniques AHE and CLAHE were applied respectively on a digital image.

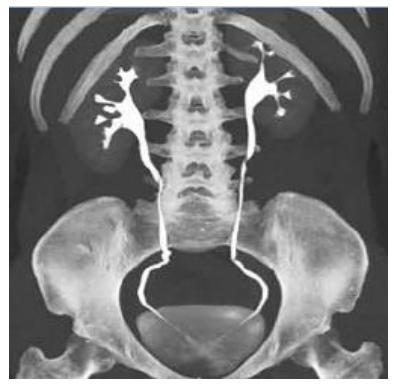

Fig. 6(a). Original image

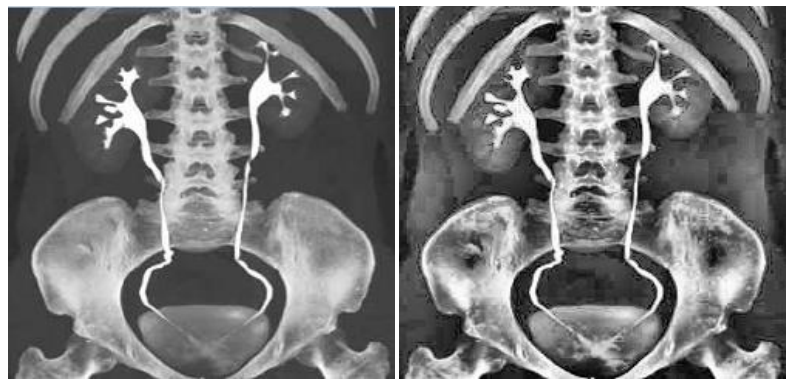

Fig. 7(a). Original image

Fig. 7(b). CLAHE image

\subsection{Parameters Used}

Table 1. Comparison of various parameters for image

\begin{tabular}{|c|c|c|c|c|}
\hline & MSE & PSNR & MEAN & SC \\
\hline HE & 882.5584 & 18.6734 & 127.4921 & 0.7397 \\
\hline AHE & 557.7013 & 20.6668 & 110.4937 & 0.9915 \\
\hline CLAHE & 833.8981 & 18.9197 & 115.6078 & 0.9239 \\
\hline
\end{tabular}

\section{CONCLUSION AND FUTURE SCOPE}

In this paper, a general review of different global and local contrast enhancement techniques is presented. Histogram equalization is a simple and effective image contrast enhancement technique. As observed in section III, histogram equalization is not suitable for consumer electronic products because it changes brightness of the image and introduces unwanted visual deterioration. Brightness preserving global enhancement techniques like BBHE and DSIHE separate the input image into two sub-images based on mean value and median value respectively. The RMSHE technique can handle higher brightness preservation than HE, BBHE and DSIHE. The RSWHE technique divides the input histogram into two or more subsections recursively, to modify sub histogram by means of weighting process based on normalized power law function. MMBEBHE is an extended version of BBHE technique and provides maximal brightness preservation comparatively. Though these global methods perform good contrast enhancement, but they do not enhance local areas of the image. Local enhancement techniques overcome this problem. AHE improves the local contrast of an image but tends to increase the noise level. CLAHE improves this by enhancing brightness to a specific range and hence facilitates the comparison of different areas of an image. In future, a combination of local and global techniques can be presented as a new idea of enhancing the images. In addition, low pass filters, high pass filters and some other enhancement filters can be used for achieving much better results.

\section{REFERENCES}

[1] Kotkar V. A. and Gharde S. S., "review of various image contrast enhancement techniques" International Journal of Innovative Research in Science, Engineering and Technology Vol. 2, Issue 7, July 2013.

[2] Rafael C. Gonzalez, and Richard E. Woods, "Digital Image Processing”, 2nd edition, Prentice Hall, 2002.

[3] Kim Y.-T., "contrast enhancement using brightness preserving Bi-Histogram equalization", IEEE Trans. Consumer Electronics, vol. 43, no. 1, pp. 1-8, Feb. 1997.

[4] Ibrahim H., and Kong N. S. P., "Brightness preserving dynamic histogram equalization for image contrast enhancement", IEEE Trans. Consumer Electronics, vol. 53, no. 4, pp. 1752 - 1758, November 2007.

[5] D. Menotti, L. Najman, J. Facon, A.A. Araujo,"Multi Histogram Equalization Methods for Contrast Enhancement and Brightness Preserving", IEEE Trans. On Consumer Electronics, vol. 53, No. 3, Aug. 2007.

[6] Y. Wang, Q. Chen, and B. Zhang, "Image enhancement based on equal area dualistic sub-image histogram equalization method," IEEE Trans. on Consumer Electronics, vol. 45, no. 1, pp. 68-75, Feb. 1999.

[7] S.-D. Chen, A. Ramli, "Contrast enhancement using recursive mean- separate histogram equalization for scalable brightness preservation," IEEE Trans. On Consumer Electronics, vol. 49, no. 4, pp. 1301-1309, Nov. 2003.

[8] Rajesh K., Harish S., and Suman, "Comparative Study of CLAHE, DSIHE \& DHE Schemes", international journal of research in management, science \& technology vol.1, issue no. 1 .

[9] Komal Vij, Yaduvir Singh, "Enhancement of Images Using Histogram Processing Techniques", Int. J. Comp. Tech. Appl., Vol. 2 (2), 309-313.

[10] Manpreet K., Jasdeep K., Jappreet K., "Survey of Contrast Enhancement Techniques based on Histogram Equalization", (IJACSA) International Journal of Advanced Computer Science and Applications, Vol. 2, No. 7, 2011.

[11] Kumar S., Verma P., Dahiya M., Gupta A., "Presence Of Noise In Dualistic Sub-Image Histogram Equalization Technique of Image Enhancement”, International Journal of Engineering Research \& Technology (IJERT) Vol. 1 Issue 3, May - 2012.

[12] Kabir M., Al-Wadud M., Chae O., "Brightness Preserving Image Contrast Enhancement using Weighted Mixture of Global and Local Transformation Functions", The International Arab Journal of Information Technology, Vol. 7, No. 4, October 2010. 
[13] Vij K., Singh Y., “ Comparison Between Different Techniques of Image Enhancement", International Journal of VLSI and Signal Processing Applications, Vol. 1, Issue 2 , May 2011,(112- 117) ,ISSN 2231-3133.

[14] Er. Mandeep K., Er. Kiran J., Er Virender L., " Study of Image Enhancement Techniques: A Review", International Journal of Advanced Research in Computer Science and Software Engineering, Volume 3, Issue 4, April 2013, ISSN: 2277 128X.

[15] S.-D. Chen, A. Ramli, "Minimum mean brightness error bihistogram equalization in contrast enhancement," IEEE Trans. on Consumer Electronics, vol. 49, no. 4, pp. 13101319, Nov. 2003

[16] Patel O., Yogendra P. S. Maravi and Sharma S., "a comparative study of histogram equalization based image enhancement techniques for brightness preservation and contrast enhancement", Signal \& Image Processing: An International Journal (SIPIJ) Vol.4, No.5, October 2013.

[17] M. Kim and M. G. Chung, "Recursively Separated and Weighted Histogram Equalization for Brightness Preservation and Contrast Enhancement" IEEE Transactions on Consumer Electronics, vol. no. 54, no. 3 , AUGUST 2008.

[18] Dhariwal S., "Comparative Analysis of Various Image Enhancement Techniques" IJECT Vol. 2, Issue 3, Sept. 2011.

[19] Rajesh Garg, Bhawna Mittal, Sheetal Garg, "Histogram Equalization Techniques for Image Enhancement" IJECT Vol. 2, Issue 1, March 2011.

[20] Ashok Saini, Mohit Bansal, Deepak Sethi, "comparison of original image enhancement using multiple histogram techniques" ijaret, Volume 1, Issue I, Feb 2013.
[21] Pujiono, Pulung N.A, I Ketut Eddy P., Mochamad H., "color enhancement of underwater coral reef images using contrast limited adaptive histogram equalization (clahe) with rayleigh distribution" The Proceedings of The 7th ICTS, Bali, May 15th-16th, 2013.

[22] Sasi Gopalan, Madhu S Nair and Souriar Sebastian "Approximation Studies on Image Enhancement Using Fuzzy Technique" International Journal of Advanced Science and Technology, Vol. 10, pp.11-26, September, 2009.

[23] Takashi K., Kota M., and Akira T., "Modified Histogram Equalization with Variable Enhancement Degree for Image Contrast Enhancement" 2009 International Symposium on Intelligent Signal Processing and Communication Systems (ISPACS 2009) December 7-9, 2009.

[24] P. Jagatheeswari, S. Suresh Kumar and M. Rajaram, "A novel approach for contrast enhancement based on histogram equalization followed by median filter" 2009 ARPN Journal of Engineering and Applied Sciences, vol. 4, no. 7, September 2009.

[25] Nicholas Sia Pik Kong, Haidi Ibrahim, and Seng Chun Hoo, "A Literature Review on Histogram Equalization and Its Variations for Digital Image Enhancement" International Journal of Innovation, Management and Technology, Vol. 4, No. 4, August 2013.

[26] J. Alex Stark, “Adaptive Image Contrast Enhancement Using Generalizations of Histogram Equalization" IEEE transactions on image processing, vol. 9, no. 5, may 2000.

[27] Pizer S. M. et. al, "Adaptive Histogram Equalization and its variations" Computer Vision, Graphics, and Image Processing 39, 355-368 (1987). 